\title{
Outpatient Urinary-Tract-Infection-Like Symptoms: Causative Microbial Survey Utilizing Multiplex Quantitative Polymerase Chain Reaction Methodology
}

\author{
Vijay Singh* (D), Carrie Wilks, Jairus Reddy, John Granger \\ Department of Molecular Diagnostics, HealthTrackRx, Denton, Texas, USA \\ Email: *vijay.singh@healthtrackrx.com
}

How to cite this paper: Singh, V., Wilks, C., Reddy, J. and Granger, J. (2020) Outpatient Urinary-Tract-Infection-Like Symptoms: Causative Microbial Survey Utilizing Multiplex Quantitative Polymerase Chain Reaction Methodology. Advances in Infectious Diseases, 10, 26-36.

https://doi.org/10.4236/aid.2020.101003

Received: February 13, 2020

Accepted: March 13, 2020

Published: March 16, 2020

Copyright $\odot 2020$ by author(s) and Scientific Research Publishing Inc. This work is licensed under the Creative Commons Attribution International License (CC BY 4.0).

http://creativecommons.org/licenses/by/4.0/

\begin{abstract}
Urinary tract infections (UTIs) are among the most common maladies afflicting the human population globally. A wide variety of microbial pathogens are responsible for causing UTIs that are often recurrent, especially in the elderly population, resulting in an economic burden of billions of dollars annually. In this study, we present data from a large population of symptomatic UTI patients tested using a multiplexed, real time quantitative polymerase chain reaction (qRT-PCR) strategy. Our data demonstrate that instances of UTI increase with age for all the tested pathogens, with the exception of $M y$ coplasma spp. and Ureaplasma spp., which were found to be significantly higher in the younger population. Furthermore, the use of the qRT-PCR approach was also found to be effective in detecting polymicrobial UTIs. With the limitations of classical microbial culture techniques traditionally used for UTI diagnosis, we demonstrate that a rapid and comprehensive technique like RT-PCR can be an effective tool for detecting and managing UTIs.
\end{abstract}

\section{Keywords}

Urinary Tract Infections, Real Time PCR, Bacterial Infections, Mycoplasma, Ureaplasma, Polymicrobial Infections

\section{Introduction}

Globally, urinary tract infections (UTIs) are among the most common microbial infections, affecting 150 million people annually [1]. Within the United States, UTIs are the most common outpatient infections, resulting in nearly 3 million emergency room visits [2]. Taking into account the direct and indirect costs, 
UTIs are responsible for an annual economic burden of approximately $\$ 3.5$ billion. UTIs are a major cause of sepsis, a life-threatening condition which results in major organ failure, and is responsible for about $20 \%$ of all global mortalities [3]. In a multicenter study of hospitalized septic patients, a urogenital source (urosepsis) was the leading cause of sepsis, even exceeding sepsis originating from a lower respiratory tract infection [4]. Due to the recurrent nature of UTIs, especially in the elderly population, these infections contribute to increased anxiety and depression in patients and have a negative impact on the overall quality of life [5].

UTIs can be classified as lower UTIs infecting the bladder (cystitis) and urethra (urethritis), or as upper UTIs infecting the kidneys (pyelonephritis). Further, the infections are classified as uncomplicated or complicated UTIs, depending upon the absence or presence of aberrations and obstructions of the genitourinary tract. Uncomplicated UTIs are more common in young sexually active females with a history of UTI [6]. Complicated UTIs are exacerbated by neurological disease, immunosuppression, pregnancy and the presence of medical instrumentation. In the United States, indwelling catheters are responsible for the majority of complicated UTI cases [7]. Instances of UTI are positively correlated with age and are one of the most commonly diagnosed infections in an older patient population [8].

The most common infection route for the UTI causing microbes is the ascent and colonization of the urethra by the fecal microflora. Escherichia coli (especially the Uropathogenic Escherichia coli (UPEC) subtype) is the most prevalent cause of UTIs across all demographics, followed by Klebsiella pneumoniae, Staphylococcus saprophyticus, Enterococcus spp. and Streptococcus agalactiae (GBS) [6]. In case of complicated UTIs, E. coli/UPEC is the most commonly identified bacteria, followed in frequency by Enterococcus spp. [9].

In this study we present a survey of pathogens responsible for UTIs, detected in urine samples of symptomatic patients, utilizing a multiplexed quantitative real-time polymerase chain reaction (qRT-PCR) method. The collated data was distributed over a wide age range to determine the prevalence of individual UTI causing pathogens. In addition, detection of polymicrobial infections in susceptible population groups was also studied.

\section{Materials and Methods}

Nucleic Acid Extraction: Urine samples obtained from symptomatic patients, were received at the HealthTrackRx laboratory in Denton, Texas, United States. The total nucleic acid from each sample was isolated using Mag-Bind Universal Pathogen Nucleic Acid Purification kit (Omega Bio-Tek, Norcross, Georgia, United States), per manufacturer's instructions on a Firefly Nimbus 96 automated liquid handling platform (Hamilton Robotics, Reno, Nevada, United States).

Reverse Transcription and Pre-Amplification: Following total nucleic acid ex- 
traction, the RNA isolated from patient urine samples was reverse transcribed using the SuperScript IV system, per manufacturer's instructions (ThermoFisher, California, United States). Rare targets in each sample were pre-amplified according to manufacturer's instructions using custom primers provided by the manufacturer (ThermoFisher, California, United States).

Real-Time PCR Analysis: The pre-amplified nucleic acid for each urine sample was placed onto an OpenArray ${ }^{\mathrm{TM}}$ plate (containing the target primers and probes), using the AccuFill ${ }^{\mathrm{TM}}$ system. The Real-Time PCR analysis was performed on a QuantStudio 12K Flex System, using the Gene Expression program according to manufacturer instructions. All PCR primers and probes used in this study were designed, validated, and manufactured by ThermoFisher's proprietary process (ThermoFisher, California, United States).

Data Analysis: All statistical analysis was performed using Microsoft Excel. Personal identifying data for patients was not accessed while performing this study. Each patient sample was assigned a randomly generated sample identification number and only the gender and age of the sample were employed for data analysis.

\section{Results and Discussion}

In the present paper, we discuss the frequency of urinary tract pathogens across a broad population range of patients displaying UTI symptoms. 5450 patient samples from 9/1/2018 to $1 / 31 / 2019$ were collected and processed at our laboratory in Denton, Texas from symptomatic potential UTI patients. Gender breakdown of the patient samples displayed a higher representation of females (79.3\%) as compared to males (20.7\%). Age distribution of the patient demographics (Table 1) showed that a majority of samples (69.7\%) were obtained from older patients (51 years and older). Our data is in agreement with what is previously known about the etiology of UTI's, wherein females (due to their anatomy and physiology) are more predisposed to contracting an UTI as compared to males [10]. In addition, it has been demonstrated in a number of studies, that UTI instances increase with age [11].

Our data did not discriminate between uncomplicated and complicated UTIs and registered the presence or absence of a microbial species in a urine sample, employing real-time PCR. Among the pathogenic microbes, both Gram-positive and Gram-negative bacteria, and fungal species were detected in the urine samples. It was found that $E$. coli was the most prevalent pathogen detected (73.69\%). This was followed by Enterococcus sp. (22.39\%), Klebsiella sp. (11.37\%), Streptococcus agalactiae (a group B Streptococcus; 8.36\%), Proteus spp. (7.43\%),

Table 1. Distribution of patient demographics on the basis of age.

\begin{tabular}{ccccc}
\hline Age (years) & $\mathbf{0 - 2}$ & $\mathbf{3 - 1 8}$ & $\mathbf{1 9}-\mathbf{5 0}$ & $\mathbf{5 1 >}$ \\
\hline Number & 25 & 162 & 1461 & 3802 \\
$\%$ & 0.45 & 2.97 & 26.80 & 69.76 \\
\hline
\end{tabular}


Enterobacter sp. (5.27\%), Mycoplasma spp. (5.24\%), Pseudomonas aeruginosa (5.01\%), Candida spp. (4.48\%), Ureaplasma spp. (4.30\%) and Staphylococcus aureus $(4.21 \%$ ) (Figure 1 ). A total of $14.2 \%$ of the samples analyzed in this study tested negative for the presence of UTI causing pathogens.

The colonic bacterial flora is a frequent source of UTI, with $E$. coli being the most common cause of both uncomplicated and complicated forms of the infection [2]. With over $70 \%$ of our samples testing positive for the presence of $E$. coIi, similar findings were observed in our study. In a clinical setting, diagnosis of an UTI is usually based on symptoms and has a very high error rate of approximately 33\% [12]. The currently accepted "gold standard" of UTI diagnosis, microbial culture and sensitivity testing (as applicable), is burdened by shortcomings, such as delayed turnaround time (due to prolonged culture time of causative microbes), guidelines with respect to positive identification or significance of identification (non-universally-accepted number of colony forming units $/ \mathrm{ml}$ defining a "significant infection", rejection of polymicrobial growth as possible contamination, etc.) [13]. All these points can pose severe implications with regard to patient care and infection management. Thus, the use of a rapid diagnosis technique employing nucleic acid amplification test (NAAT) can preclude these possibilities and empower better clinical decisions with respect to patient care.

Among the coagulase-negative staphylococci, S. saprophyticus has been observed to be a significant source of UTIs second only to $E$. coli [14]. In our study

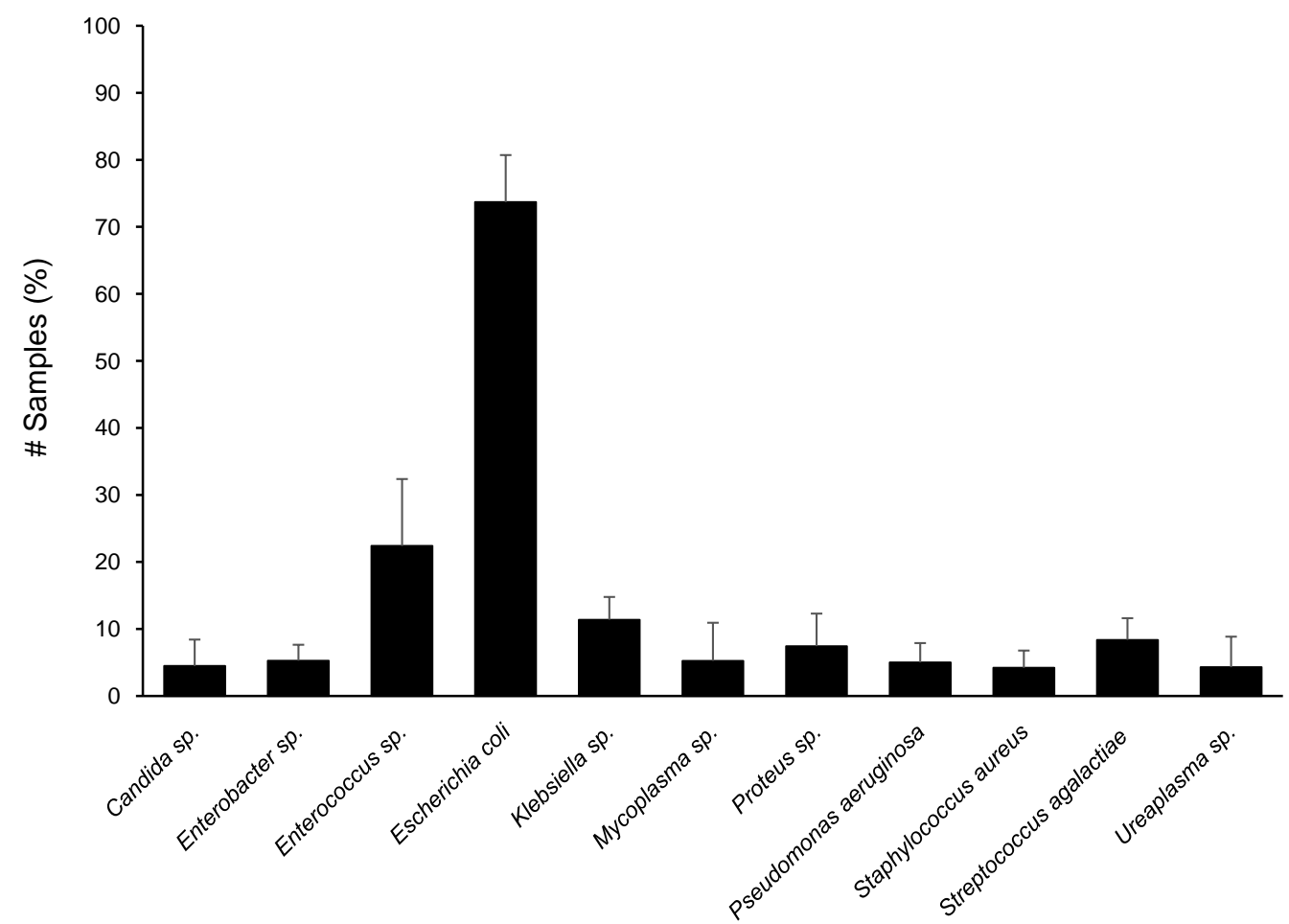

Figure 1. Prevalence (\%) of the common UTI causative agents detected in the urine samples of symptomatic patients using multiplex real-time PCR. 
we also found a significant number of samples positive for the presence of coagulase-negative staphylococcal species. However, since our lab uses a pooled assay (S. saprophyticus, S. epidermidis, S. haemolyticus, S. lugdunensis) for these targets, species-specific identification is not possible; therefore, this data was not included in the present analysis.

With $22.39 \%$ of the total samples testing positive for Enterococcus spp. (E. faecalis and E. faecium), a higher than previously reported level of Enterococcus infection was detected in this study. The emergence of $E$. faecium as a prominent nosocomial pathogen within the last two decades, due to its multi-drug resistant nature and overuse of antibiotics, poses a significant global challenge to healthcare practitioners. Within the United States, the early 2000s saw an almost two-fold increase in vancomycin resistant Enterococcus (VRE) infections in hospitals [15], and a perceptible shift in the principal pathogen from E. faecalis to E. faecium, with the latter displaying more vancomycin resistant isolates [16]. Enterococci can survive for an extended period in an exposed environment, which is possible due to their resistance to a number of physical and chemical decontaminants. This allows for these pathogens to persist and patients to acquire hospital acquired infections [17].

With respect to the levels of other established etiological agents of UTI, our study did not find a significant difference when compared to previously published results [18]. For example, Proteus spp. ( $P$. mirabilis and $P$. vulgaris) and $S$. aureus were, respectively, detected in $7.43 \%$ and $4.21 \%$ of the total tested samples (Figure 1). Proteus mirabilis is a Gram-negative member of the Enterobacteriaceae, which can result in both cystitis and pyelonephritis, especially in elderly patients [19]. This pathogen is responsible for $1 \%-10 \%$ of UTI's, and up to $44 \%$ of complicated catheter-associated UTI's [20]. Among the nosocomial pathogens responsible for UTI's, $S$. aureus is considered atypical and is responsible for nearly $6 \%$ of the infections. Bacteriuria caused by $\mathcal{S}$. aureus may be a result of catheterization, or it may take a hematogenous route due to an undetected bacteremia [21]. It has been demonstrated that $S$. aureus bacteriuria results in higher mortality in patients [22], with the severity increased in case of methicillin resistant $S$. aureus (MRSA) urogenital infections [23].

Distribution of individual pathogens detected, according to age, revealed a pattern that reinforces the observation that UTI instances become more prevalent with advancing age (Figure 2). All of the pathogens, with the exception of Mycoplasma spp. and Ureaplasma spp., were detected at a higher rate $(69.9 \% \pm$ $7.4 \%$ ) in the older population (51 years of age and older), followed by the middle-aged population (19 - 50 years of age), with an average detection rate of $27.6 \% \pm 6.9 \%$ (Figure 2 and Figure 3). Interestingly, Mycoplasma spp. ( $M$. genitalium and $M$. hominis) and Ureaplasma spp. (U. urealyticum and $U$. parvum) were detected at a significantly higher rate $(73.88 \%$ and $72.15 \%$ respectively) in the 19 - 50 years of age patients and at a lower rate $(16.11 \%$ and $18.98 \%$ respectively) in the older (51 years and older) patient population (Figure 3). 

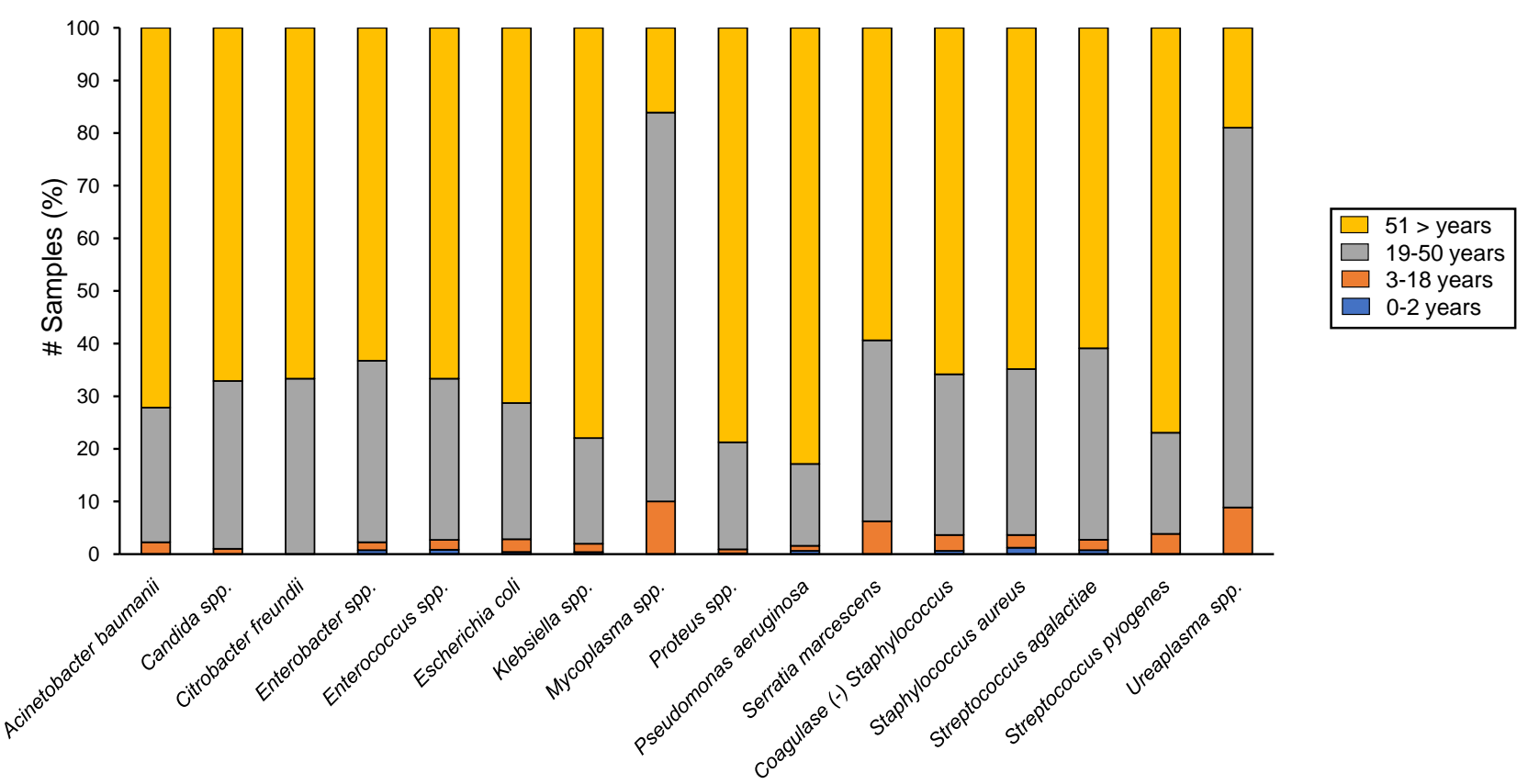

Figure 2. Distribution of individual UTI causing pathogens according to age. The patient population was divided into 4 age groups: 0 - 2 years $(n=25), 3-18$ years $(n=162), 19-50$ years $(n=1461)$ and 51 years and older $(n=3802)$.

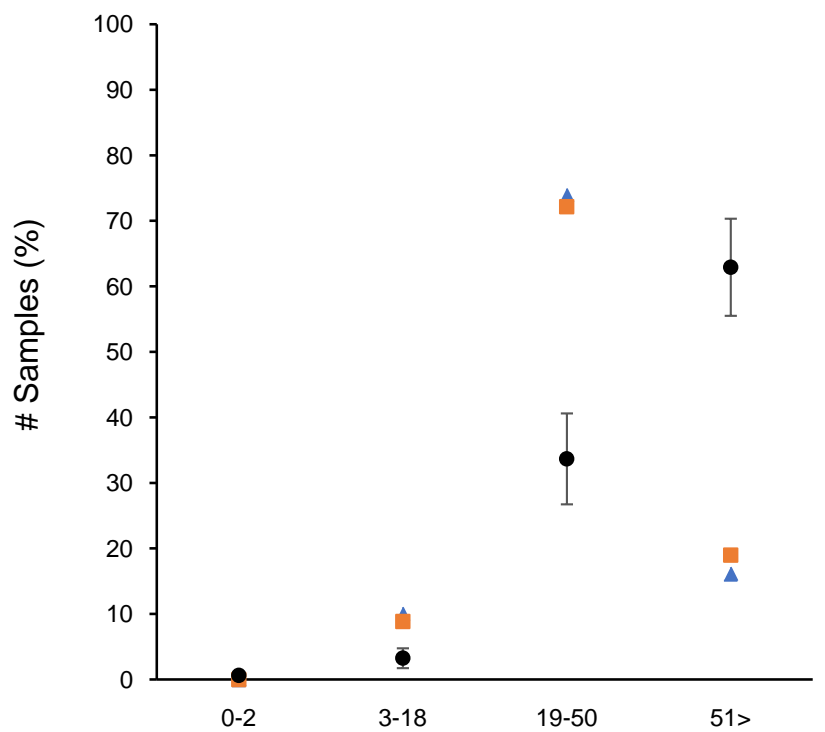

Figure 3. Instances of UTI are directly correlated with age. Mycoplasma spp. and Ureaplasma spp. display an exceptional distribution trend wherein these pathogens display a higher detection rate in the younger population group (19 - 50 years of age) when compared to the other detected UTI pathogens in the same age group. Error bars represent standard deviation.

Members of the Mollicutes class represent a unique class of parasitic/saprophytic organisms that are defined by their lack of cell wall, small genome size, and consequently a limited anabolic capacity. M. genitalium, M. hominis, U. urealyticum, and $U$. parvum are the most common mycoplasma species that are responsible for UTIs in both men and women resulting in urethritis, cystitis, and bladder 
pain [24]. The instances of Mycoplasma spp. and Ureaplasma spp. infections have been found to be directly proportional to the number of sexual partners and activity in the previous 2 months to diagnosis. Detection of these pathogens by a rapid NAAT methodology gains importance since classical culture methods can take 2 - 3 weeks before a positive identification can be made [25]. Our data bolster this observation, with the detection frequency of these pathogens being significantly higher in our study's sexually active population (Figure 3).

Clinical microbiology testing that involves the use of traditional culturing techniques for the detection of UTIs can prove to be inadequate in providing the complete picture of the etiological agents responsible for the infection. It has been estimated that nearly $33 \%$ of urine cultures from elderly patients are polymicrobial. However, due to the inherent difficulty in identifying the individual organisms in a mixed culture, laboratories do not always report all potentially pathogenic bacteria [26].

Among the urine samples analyzed in this study, a majority was from patients suffering from chronic kidney disease (CKD) (55.1\%) or from obstetric/gynecological facilities (20.3\%). A review of published literature revealed very sparse information on the status of UTI in CKD patients [27] [28]. No significant difference was observed between the CKD patient and the overall population samples for the detection of pathogens (Figure 4). It has been previously suggested that, adjusted for age, CKD patients do not display an increased risk of UTIs when compared to the general population [27]. It has been documented that UTI instances are not significantly higher in pregnant women when compared to non-pregnant women, However, the management of these infections in the pregnant population becomes complicated, due to the risks posed to the unborn child [29]. UTIs during pregnancy can potentially result in increased risk of pre-eclampsia, pre-mature birth, low neonatal birth weight, and miscarriage

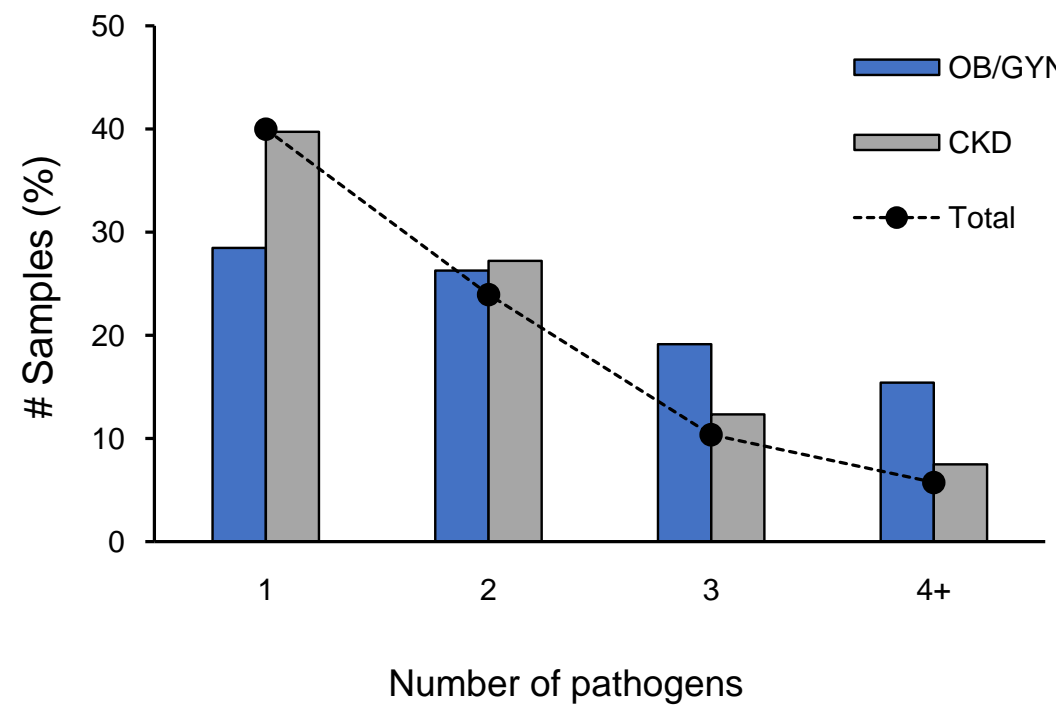

Figure 4. Distribution of mono- and polymicrobial infections in urine samples of chronic kidney disease (CKD) patients and pregnant women (OB/GYN). 
[30]. Mycoplasma spp. and Ureaplasma spp. are the most important preventable causes of female infertility and adverse pregnancy outcomes [31]. Due to significant maternal colonization or infection, and subsequent fetal transmission, a growing body of evidence supports pre-natal diagnosis (preferably by a NAAT methodology) and treatment of these microbes [32] [33] We found that detection of polymicrobial infections ( 3 or more pathogens) in the urine samples of the pregnant population was significantly higher than the overall detection rate (Figure 4).

A recent study of symptomatic UTI patients demonstrated that multiplex PCR was able to detect the presence of pathogenic agents in 36\% of concurrent, parallel "split samples", that tested negative with traditional culture techniques [34]. In addition, the same study detected polymicrobial infections in $12 \%$ of culture negative samples. Although a direct comparison with culture was not performed on the samples used in the present study, our data suggest that PCR can be successfully used for the detection of polymicrobial urinary tract infections in high-risk population groups, like pregnant women.

In summary, our data support the previously published observations that UTI instances increase with age, and that E. coli/UPEC is the single most prevalent infectious agent. However, we observed a higher than previously reported level of Enterococcus spp. infection. With an aging population, the management of UTIs gains significance with respect to economic and healthcare perspective. We report a significantly higher detection of Mycoplasma spp. and Ureaplasma spp. in the younger, sexually active population when compared to other UTI causing agents, suggesting that this population group should be actively screened for these pathogens. Classical microbial culture is still considered to be the gold standard of UTI diagnosis, but a growing body of evidence (including our current data) shows that these standards should be updated to reflect the comparative superiority of NAAT-based infectious disease testing. Examples of culture limitations include the facts that polymicrobial (especially when 3 or more microbes are present) cultures are often dismissed as contamination, or that fastidious, difficult-to-culture organisms (Mycoplasma spp. and Ureaplasma spp.) can take up to 8 weeks to culture. Significantly, the use of a NAAT technique, like qRT-PCR, can provide unambiguous UTI diagnosis with a rapid turnaround time, thereby improving the quality of clinical decisions in the management of urogenital infections. The present study was limited in its scope as we are only reporting the detection of etiologic agents in the urine samples of symptomatic patients. We did not have access to the patient history, prior infection reports, antibiotic use, etc. As a future endeavor, further analysis of UTIs, with these features incorporated is planned.

\section{Conflicts of Interest}

The authors declare no conflicts of interest regarding the publication of this paper. 


\section{References}

[1] Stamm, W.E. and Norrby, S.R. (2001) Urinary Tract Infections: Disease Panorama and Challenges. The Journal of Infectious Diseases, 183, S1-S4.

https://doi.org/10.1086/318850

[2] Foxman, B. (2014) Urinary Tract Infection Syndromes: Occurrence, Recurrence, Bacteriology, Risk Factors, and Disease Burden. Infectious Disease Clinics of North America, 28, 1-13. https://doi.org/10.1016/j.idc.2013.09.003

[3] Rudd, K.E., Johnson, S.C., Agesa, K.M., Shackelford, K.A., Tsoi, D., Kievlan, D.R. and Fleischmann-Struzek, C. (2020) Global, Regional, and National Sepsis Incidence and Mortality, 1990-2017: Analysis for the Global Burden of Disease Study. The Lancet, 395, 200-211. https://doi.org/10.1016/S0140-6736(19)32989-7

[4] Chou, E.H., Mann, S., Hsu, T.C., Hsu, W.T., Liu, C.C.Y., Bhakta, T. and Lee, C.C. (2020) Incidence, Trends, and Outcomes of Infection Sites among Hospitalizations of Sepsis: A Nationwide Study. PLoS ONE, 15, e0227752. https://doi.org/10.1371/journal.pone.0227752

[5] Renard, J., Ballarini, S., Mascarenhas, T., Zahran, M., Quimper, E., Choucair, J. and Iselin, C.E. (2015) Recurrent Lower Urinary Tract Infections Have a Detrimental Effect on Patient Quality of Life: A Prospective, Observational Study. Infectious Diseases and Therapy, 4, 125-135. https://doi.org/10.1007/s40121-014-0054-6

[6] Foxman, B. (2010) The Epidemiology of Urinary Tract Infection. Nature Reviews Urology, 7, 653-660. https://doi.org/10.1038/nrurol.2010.190

[7] Lo, E., Nicolle, L.E., Coffin, S.E., Gould, C., Maragakis, L.L., Meddings, J. and Yokoe, D.S. (2014) Strategies to Prevent Catheter-Associated Urinary Tract Infections in Acute Care Hospitals: 2014 Update. Infection Control and Hospital Epidemiology, 35, 464-479. https://doi.org/10.1086/675718

[8] Tsan, L., Langberg, R., Davis, C., Phillips, Y., Pierce, J., Hojlo, C. and Danko, L. (2010) Nursing Home-Associated Infections in Department of Veterans Affairs Community Living Centers. American Journal of Infection Control, 38, 461-466. https://doi.org/10.1016/j.ajic.2009.12.009

[9] Levison, M.E. and Kaye, D. (2013) Treatment of Complicated Urinary Tract Infections with an Emphasis on Drug-Resistant Gram-Negative Uropathogens. Current Infectious Disease Reports, 15, 109-115. https://doi.org/10.1007/s11908-013-0315-7

[10] Abelson, B., Sun, D., Que, L., Nebel, R.A., Baker, D., Popiel, P. and Fraser, M.O. (2018) Sex Differences in Lower Urinary Tract Biology and Physiology. Biology of Sex Differences, 9, 1-13. https://doi.org/10.1186/s13293-018-0204-8

[11] Rowe, T.A. and Juthani-Mehta, M. (2013) Urinary Tract Infection in Older Adults. Aging Health, 9, 519-528. https://doi.org/10.2217/ahe.13.38

[12] Schmiemann, G., Kniehl, E., Gebhardt, K., Matejczyk, M.M. and Hummers-Pradier, E. (2010) The Diagnosis of Urinary Tract Infection: A Systematic Review. Deutsches Ärzteblatt International, 107, 361. https://doi.org/10.3238/arztebl.2010.0361

[13] Hooton, T.M., Bradley, S.F., Cardenas, D.D., Colgan, R., Geerlings, S.E., Rice, J.C. and Nicolle, L.E. (2010) Diagnosis, Prevention, and Treatment of Catheter-Associated Urinary Tract Infection in Adults: 2009 International Clinical Practice Guidelines from the Infectious Diseases Society of America. Clinical Infectious Diseases, 50, 625-663. https://doi.org/10.1086/650482

[14] Hovelius, B. and Mårdh, P.A. (1984) Staphylococcus saprophyticus as a Common Cause of Urinary Tract Infections. Reviews of Infectious Diseases, 6, 328-337. https://doi.org/10.1093/clinids/6.3.328 
[15] Ramsey, A.M. and Zilberberg, M.D. (2009) Secular Trends of Hospitalization with Vancomycin-Resistant Enterococcus Infection in the United States, 2000-2006. Infection Control and Hospital Epidemiology, 30, 184-186. https://doi.org/10.1086/593956

[16] Arias, C.A. and Murray, B.E. (2008) Emergence and Management of Drug-Resistant Enterococcal Infections. Expert Review of Anti-Infective Therapy, 6, 637-655. https://doi.org/10.1586/14787210.6.5.637

[17] Bradley, C.R. and Fraise, A.P. (1996) Heat and Chemical Resistance of Enterococci. Journal of Hospital Infection, 34, 191-196. https://doi.org/10.1016/S0195-6701(96)90065-1

[18] Flores-Mireles, A.L., Walker, J.N., Caparon, M. and Hultgren, S.J. (2015) Urinary Tract Infections: Epidemiology, Mechanisms of Infection and Treatment Options. Nature Reviews Microbiology, 13, 269-284. https://doi.org/10.1038/nrmicro3432

[19] Matthews, S.J. and Lancaster, J.W. (2011) Urinary Tract Infections in the Elderly Population. The American Journal of Geriatric Pharmacotherapy, 9, 286-309. https://doi.org/10.1016/j.amjopharm.2011.07.002

[20] Jacobsen, S.M., Stickler, D.J., Mobley, H.L.T. and Shirtliff, M.E. (2008) Complicated Catheter-Associated Urinary Tract Infections Due to Escherichia coli and Proteus mirabilis. Clinical Microbiology Reviews, 21, 26-59. https://doi.org/10.1128/CMR.00019-07

[21] Baraboutis, I.G., Tsagalou, E.P., Lepinski, J.L., Papakonstantinou, I., Papastamopoulos, V., Skoutelis, A.T. and Johnson, S. (2010) Primary Staphylococcus aureus Urinary Tract Infection: The Role of Undetected Hematogenous Seeding of the Urinary Tract. European Journal of Clinical Microbiology and Infectious Diseases, 29, 1095-1101. https://doi.org/10.1007/s10096-010-0967-2

[22] Huggan, P.J., Murdoch, D.R., Gallagher, K. and Chambers, S.T. (2008) Concomitant Staphylococcus aureus Bacteremia Is Associated with Poor Clinical Outcome in Adults with $S$. aureus Bacteraemia. Journal of Hospital Infection, 69, 345-349. https://doi.org/10.1016/j.jhin.2008.04.027

[23] Shurland, S., Zhan, M., Bradham, D.D. and Roghmann, M.C. (2007) Comparison of Mortality Risk Associated with Bacteremia Due to Methicillin-Resistant and Methicillin-Susceptible Staphylococcus aureus. Infection Control and Hospital Epidemiology, 28, 273-279. https://doi.org/10.1086/512627

[24] Combaz-Söhnchen, N. and Kuhn, A. (2017) A Systematic Review of Mycoplasma and Ureaplasma in Urogynaecology. Geburtshilfe und Frauenheilkunde, 77, 1299-1303. https://doi.org/10.1055/s-0043-119687

[25] Baseman, J.B., Cagle, M., Korte, J.E., Herrera, C., Rasmussen, W.G., Baseman, J.G. and Piper, J.M. (2004) Diagnostic Assessment of Mycoplasma genitalium in Culture-Positive Women. Journal of Clinical Microbiology, 42, 203-211. https://doi.org/10.1128/JCM.42.1.203-211.2004

[26] Cove-Smith, A. and Almond, M.K. (2007) Management of Urinary Tract Infections in the Elderly. Trends in Urology, Gynaecology and Sexual Health, 12, 31-34. https://doi.org/10.1002/tre.33

[27] Gilbert, D.N. (2006) Urinary Tract Infections in Patients with Chronic Renal Insufficiency. Clinical Journal of the American Society of Nephrology, 1, 327-331. https://doi.org/10.2215/CJN.01931105

[28] Hsiao, C.Y., Lin, H.L., Lin, Y.K., Chen, C.W., Cheng, Y.C., Lee, W.C. and Wu, T.C. (2014) Urinary Tract Infection in Patients with Chronic Kidney Disease. Turkish Journal of Medical Sciences, 44, 145-149. https://doi.org/10.3906/sag-1303-51 
[29] Schnarr, J. and Smaill, F. (2008) Asymptomatic Bacteriuria and Symptomatic Urinary Tract Infections in Pregnancy. European Journal of Clinical Investigation, 38, 50-57. https://doi.org/10.1111/j.1365-2362.2008.02009.x

[30] Gravett, C.A., Gravett, M.G., Martin, E.T., Bernson, J.D., Khan, S., Boyle, D.S. and Steele, M.S. (2012) Serious and Life-Threatening Pregnancy-Related Infections: Opportunities to Reduce the Global Burden. PLoS Medicine, 9, e1001324. https://doi.org/10.1371/journal.pmed.1001324

[31] Ljubin-Sternak, S. and Meštrović, T. (2014) Chlamydia Trachomatis and Genital Mycoplasmas: Pathogens with an Impact on Human Reproductive Health. Journal of Pathogens, 2014, Article ID: 183167. https://doi.org/10.1155/2014/183167

[32] Oeser, C., Pond, M., Butcher, P., Bedford Russell, A., Henneke, P., Laing, K. and Harris, K. (2020) PCR for the Detection of Pathogens in Neonatal Early Onset Sepsis. PLoS ONE, 15, e0226817. https://doi.org/10.1371/journal.pone.0226817

[33] Waites, K.B., Katz, B. and Schelonka, R.L. (2005) Mycoplasmas and Ureaplasmas as Neonatal Pathogens. Clinical Microbiology Reviews, 18, 757-789. https://doi.org/10.1128/CMR.18.4.757-789.2005

[34] Wojno, K.J., Baunoch, D., Luke, N., Opel, M., Korman, H., Kelly, C. and Makhloouf, B. (2020) Multiplex PCR Based Urinary Tract Infection (UTI) Analysis Compared to Traditional Urine Culture in Identifying Significant Pathogens in Symptomatic Patients. Urology, 136, 119-126.

https://doi.org/10.1016/j.urology.2019.10.018 